\title{
TWO-STATION METEOR OBSERVATIONS WITH MINI-MEGATORTORA AND FAVOR WIDE-FIELD MONITORING SYSTEMS
}

\author{
S. Karpov ${ }^{1,2,4}$, N. Orekhova ${ }^{3}$, G. Beskin ${ }^{2,4}$, A. Biryukov ${ }^{5,4}$, S. Bondar ${ }^{3}$, E. Ivanov ${ }^{3}$, E. Katkova ${ }^{3}$, A. Perkov ${ }^{3}$, \\ V. Plokhotnichenko ${ }^{2}$, and V. Sasyuk ${ }^{4}$
}

\begin{abstract}
Here we present the results of our four years long observations of meteors with Mini-MegaTORTORA wide-field monitoring system with sub-second temporal resolution. Over this period, we detected and catalogued more than 175000 faint meteors with magnitudes down to 8-10 mag and angular velocities up to $40 \mathrm{deg} / \mathrm{s}$. Recently, we started double-station observations using Mini-MegaTORTORA together with reconstructed FAVOR camera on $3.8 \mathrm{~km}$ baseline. This setup allows to observe tens of faint meteors per night. We present the preliminary results of such observations.
\end{abstract}

\section{RESUMEN}

Presentamos los resultados de las observaciones de meteoros realizadas durante 4 años con MiniMegaTORTORA, un sistema con óptica de gran campo con el que conseguimos resolución sub-segundo. Durante este tiempo, detectamos más de 175.000 meteoros con magnitudes en el rango 8-10 y velocidades angulares de hasta 40 grados $\mathrm{s}^{-1}$. Recientemente hemos empezado observaciones en doble estación conjuntamente con la reconstruida cámara FAVOR a 3,8 km de distancia. Este sistema permite detectar decenas de meteoros débiles cada noche. Mostramos los resultados preliminares de este estudio.

Key Words: astronomical databases: miscellaneous — meteorites, meteors, meteoroids

\section{INTRODUCTION}

The database of the IAU Meteor Data Center (Jopek \& Jenniskens 2011; Jopek \& Kan̆uchová 2014) contains information about 956 meteor showers (as of January 2018), with only 112 of them listed as "established", i.e. confirmed. The other 819 are in the "working list" - these are showers whose parameters are not derived or confirmed after first observations. Promotion to the list of established ones requires a number of confirmation detections, as well as a two-stations observations to estimate their velocities and directions and derive their orbits inside Solar system.

Nowadays, the video observations of meteors are typically employed to solve this problem. However, due to the application of fish-eye cameras with high frame rates and low angular resolution, such observations allows the detection of the brightest events and fireballs only. On the other hand, a lot of ex-

\footnotetext{
${ }^{1}$ CEICO, Institute of Physics, Czech Academy of Sciences, Prague, Czech Republic.

${ }^{2}$ Special Astrophysical Observatory of Russian Academy of Sciences, Russia.

${ }^{3}$ Research and Production Corporation "Precision Systems and Instruments", Russia.

${ }^{4}$ Kazan Federal University, Russia.

${ }^{5}$ Moscow State University, Russia.
}

periments are systematically monitoring the sky using wide-field telescopes with narrower fields of view in order to solve various astrophysical tasks, from searching for variable stars, gamma-ray bursts and supernovae to detecting potentially hazardous asteroids, and while not specifically optimized for meteor observations, some of them may still provide a lot of information on meteors, especially if their temporal resolution is better than a second. The best examples of such systems are the Mini-MegaTORTORA

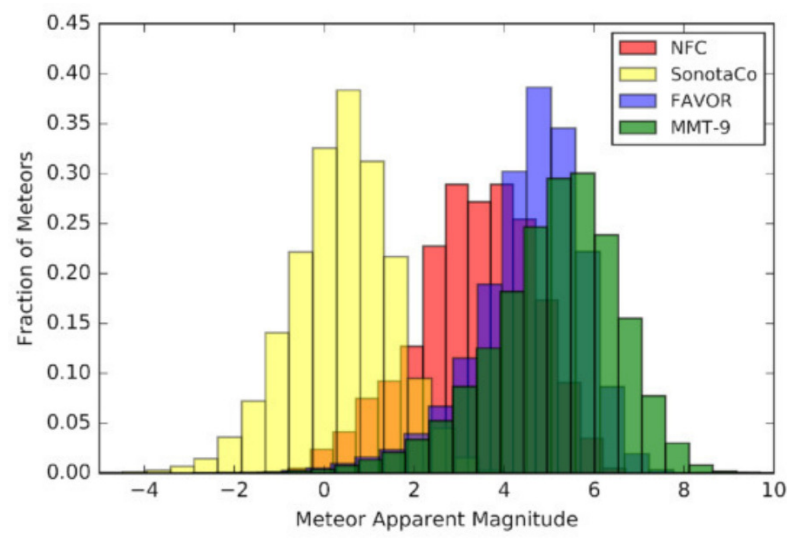

Fig. 1. Comparison of typical meteor brightnesses from several experiments and public databases. 

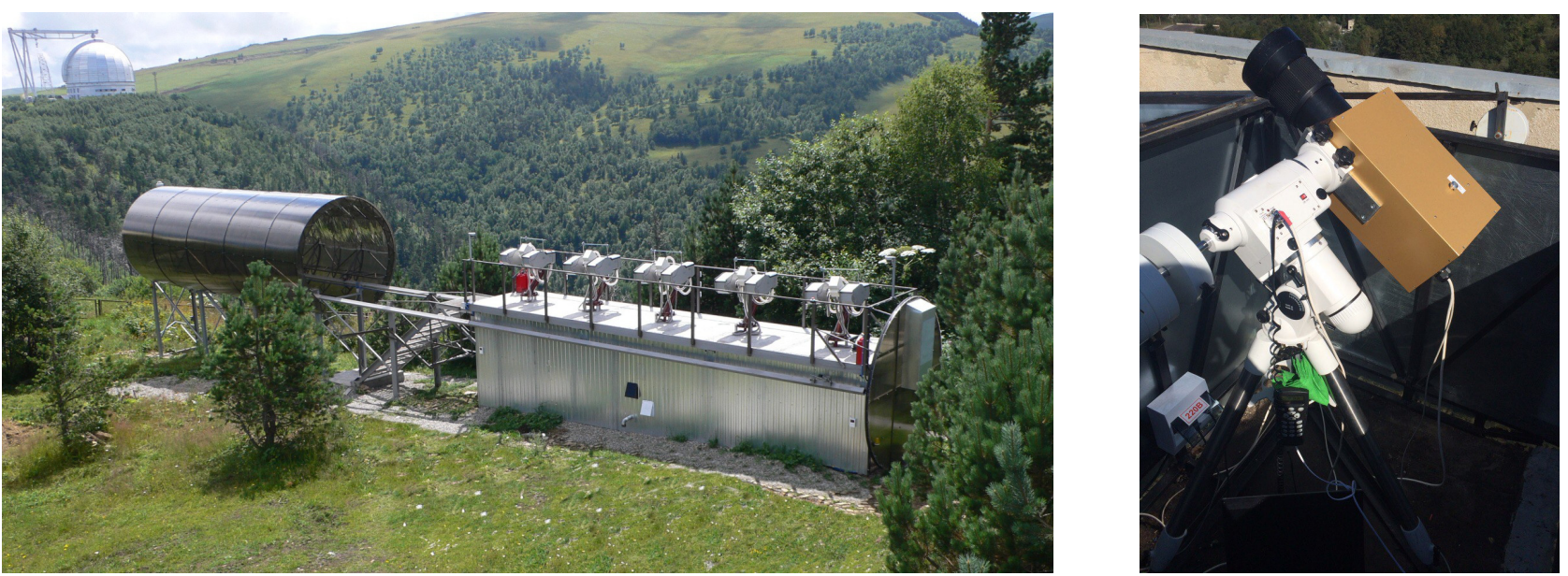

Fig. 2. The hardware used for a two-station meteor observations. Left - Mini-MegaTORTORA multi-channel system, located near Russian 6-m telescope of Special Astrophysical Observatory. Right - reconstructed FAVOR camera, located on the roof of main building of Special Astrophysical Observatory, at $3.8 \mathrm{~km}$ distance from Mini-MegaTORTORA.

multichannel instrument which is operational now, and the historical FAVOR and TORTORA cameras, all created and operated by our group. Their combination of quite large fields of view (hundreds of square degrees), sufficiently high temporal resolution (up to 10 frames per second) and deep detection limit (down to 10-11 mag on the shortest timescales) make them perfect instruments for studying meteors at least several magnitudes fainter than the ones typically seen by video systems, and therefore to systematically study the lower-mass component of meteoroid streams in order to recover their complete distribution over all ranges of sizes and masses.

In this article we present the results of our study of such faint meteors using Mini-MegaTORTORA wide-field monitoring system, as well as our recent efforts of performing two-station observations using it in combination with reconstructed FAVOR camera.

\section{MINI-MEGATORTORA AND FAVOR CAMERAS}

The Mini-MegaTORTORA (MMT-9) is a 9channel monitoring system with near 900 square degrees field and a temporal resolution of 0.1 seconds, the magnitude limit down to $m_{V}=11$ for stars, and $16^{\prime \prime}$ pixel size (Beskin et al. 2013, 2014, 2017; Karpov et al. 2017a). It is in operation at Special Astrophysical Observatory, Russia, since mid-2014, and now continuously monitors the sky looking for fast optical transients of various nature, from artificial satellites (Karpov et al. 2016a) to gamma-ray bursts (Karpov et al. 2017b). Its real-time transient detection system routinely extracts meteors from the data stream using their elongated shape, and then analyzes them automatically to derive their brightness along the trails, the light curves, the trajectories, angular velocities and durations, as well as colors if the event is observed by several channels with photometric filters installed. All this information is then stored to the publicly accessible database (Karpov et al. 2016b) available online ${ }^{6}$.

The database also contains the same information for 10117 meteors observed with the historical FAVOR camera (Zolotukhin et al. 2004; Karpov et al. 2005, 2010) during 2006-2009, uniformly processed with the same software used for MiniMegaTORTORA data analysis now. Figure 1 shows the comparison of peak magnitudes (integral brightness of the meteor trail on a single frame during the maximum brightness of the meteor) of events observed with Mini-MegaTORTORA and FAVOR, as well as meteors from SonotaCo (2009) database and the ones observed with NFC (Koukal et al. 2015) cameras.

Original FAVOR hardware had been severely damaged due to thunderstorm back in 2009, and it had not been operational since then. However, in mid-2017 we revived and modernized it in order to implement two-station regime of meteor observations together with Mini-MegaTORTORA. The presently reconstructed FAVOR camera is placed on the roof of the main building of Special Astrophysical Observatory, Russia, at $3.8 \mathrm{~km}$ distance from

\footnotetext{
${ }^{6}$ The database is published at http://mmt.favor2.info/ meteors and http://astroguard.ru/meteors, and contains, as of Jan 2018, more than 187000 events observed by MiniMegaTORTORA in 2014-2018
} 


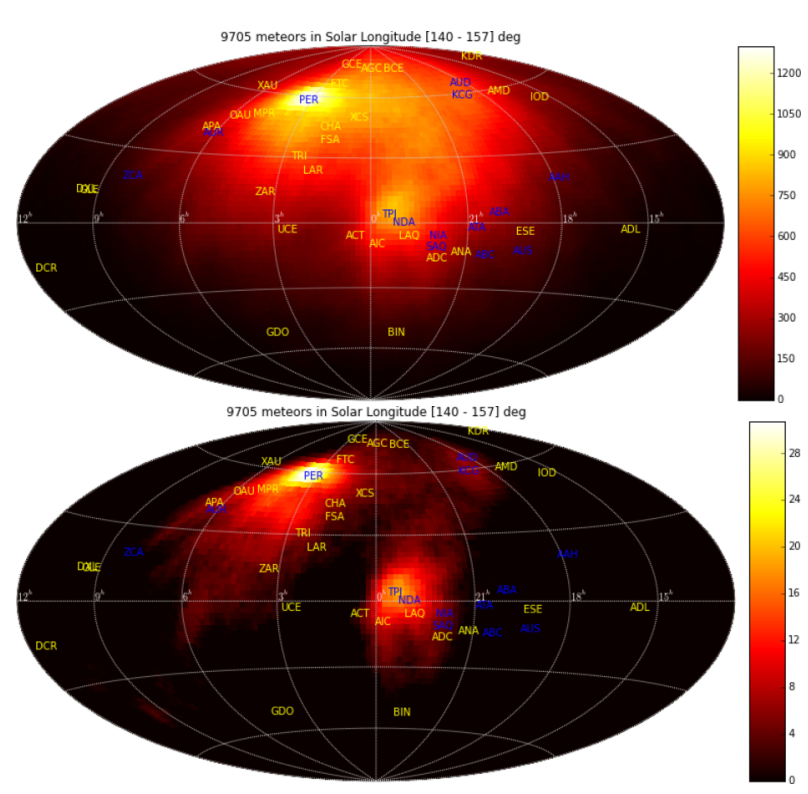

Fig. 3. Example of a statistical radiant determination using meteors from Mini-MegaTORTORA database around annual Perseid activity interval. Upper panel unnormalized map, measured in number of events potentially passing close to (originating from) a given position on the sky. Lower panel - the same corrected for the sky coverage of actual observations, and normalized by the variance of simulated sky maps having random orientation of meteors.

Mini-MegaTORTORA. The field of view of FAVOR is now $\sim 600$ square degrees, temporal resolution 0.13 $\mathrm{s}$ and stellar detection limit $m_{V} \sim 9$ mag. Detection and analysis pipeline is the same as used for MiniMegaTORTORA (see Figure 2).

\section{STATISTICAL DETERMINATION OF SHOWER RADIANTS}

The astrometric accuracy of MiniMegaTORTORA $(\sim 15$ arcsec/pix $)$ and FAVOR $(\sim 60 \mathrm{arcsec} / \mathrm{pix})$ are perfectly enough for detecting meteor parallaxes $(\sim 2$ degrees at $100 \mathrm{~km})$ on $\sim 3.8 \mathrm{~km}$ baseline, and estimating the apparent radiant positions using the method of great circle intersection. The accuracy of such determination is better than a few degrees for a larger part of events depending on trail orientation relative to the basis.

Even with a single-station data, like most of events contained in Mini-MegaTORTORA online database, it is still possible to derive radiant positions of meteor streams by means of statistical methods. Indeed, every night Mini-MegaTORTORA detects hundreds of meteor events, and the intersections of their trails on the sky should reveal positions of radiants active on that night. We implemented an

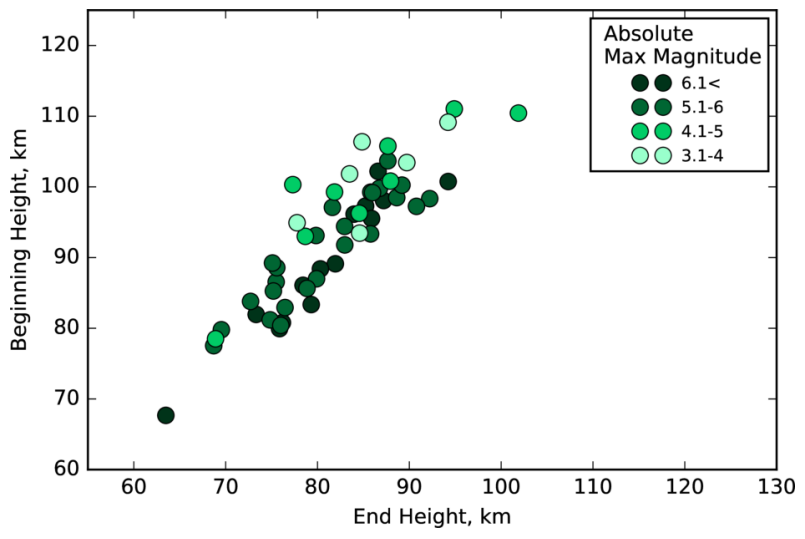

Fig. 4. Dependence of beginning and end atmospheric heights of meteor events on absolute maximum magnitude, derived from two-station observations.

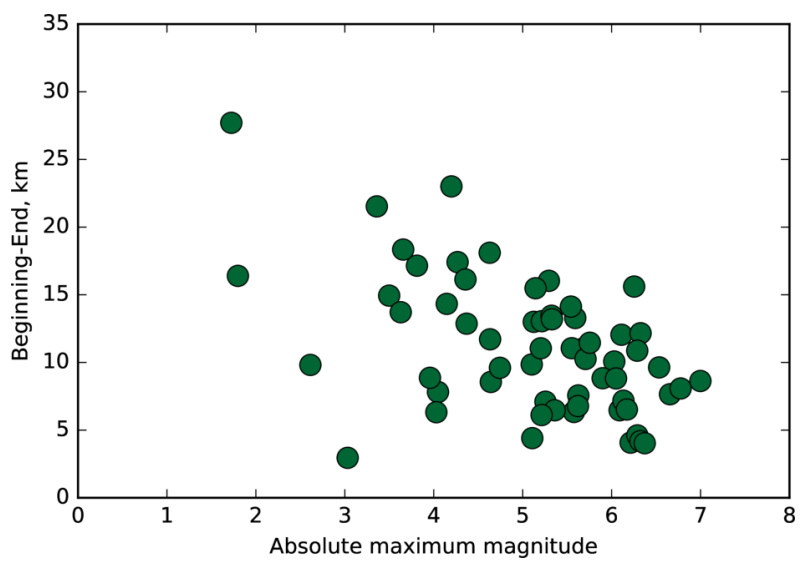

Fig. 5. Dependence of meteor height loss on absolute maximum magnitude, derived from two-station observations.

even simpler and faster method for such estimation which scales linearly with the number of meteors as it does not require implicit computation of pairwise intersections of individual trails. The method consists of building a coarse grid on the sky and computing the number of meteor trails (more precisely - the continuations of meteor trails along great circles 90 degrees back from their heads) passing inside a pre-defined distance, e.g. 2 degrees, from every grid points. The resulting map has to be then normalized to account for quite small field of view of Mini-MegaTORTORA system which simultaneously see only $\sim 1 / 20$ part of hemisphere. To do it, we repeatedly simulate the series of artificial meteor events having the same sky positions as real ones, but with random orientations, and compute the mean and variance maps by the same method. Finally, we may subtract the mean value from actual map and divide by variance, and therefore get 
an estimate of radiant significance at every position on the sky. An example of such map is given in Figure 3, while the animation of an annual meteor activity cycle according to Mini-MegaTORTORA is available online ${ }^{7}$.

\section{TWO-STATION OBSERVATIONS OF METEORS}

For 10 nights in Aug 2017 we performed test two-station observations of meteors using MiniMegaTORTORA and FAVOR cameras. After extraction of meteors by real-time transient detection system, the events from two instruments have been matched using temporal and spatial proximity, as well as similarity in the trail orientation on the sky (which is necessary, as often Mini-MegaTORTORA detects several different meteor events nearly simultaneously due to its wide field of view and deep detection limit). Then, the events completely inside Mini-MegaTORTORA field of view (i.e. with both beginning and end of trail visible) have been selected, and their atmospheric heights have been determined using geometrical parallax method, similar to the one used by Golubaev (2015). Using spherical trigonometric equations we estimated the parallactic angles for appatent meteor positions, and then determined its distance from Mini-MegaTORTORA, and finally - its height over the Earth ellipsoid (Vallado 2007).

For 40 meteors we successfully derived the heights of their appearance and disappearance (beginning and end of the trail), as well as peak absolute magnitudes (see Figures 4 and 5). Despite quite small distance between two stations, the accuracy of height determination is between $3 \%$ and $11 \%$, depending on the relative orientation of meteor trail in respect to the baseline.

In the future we plan to start measuring linear velocities of meteors which will allow to derive their orbits. Trigonometric estimations alone do not provide sufficient accuracy for it.

Acknowledgements: Mini-MegaTORTORA belongs to Kazan Federal University and the work is performed according to the Russian Government
Program of Competitive Growth of Kazan Federal University. Observations on Mini-MegaTORTORA are supported by the Russian Science Foundation grant No. 14-50-00043. The reported study was funded by RFBR according to the research project No. 17-52-45048. This work was supported by European Structural and Investment Fund and the Czech Ministry of Education, Youth and Sports (Project CoGraDS - CZ.02.1.01/0.0/0.0/15_003/0000437).

\section{REFERENCES}

Beskin, G. M., Karpov, S. V., Plokhotnichenko, V. L., et al. 2013, PhyU, 56, 836

Beskin, G., Karpov, S., Bondar, S., et al. 2014, RMxAC, 45,20

Beskin, G., Biryukov, A., Bondar, E., et al. 2017a, ASPC, 510,526

Beskin, G. M., Karpov, S. V., Biryukov, A. V., et al. 2017, AstBu, 72, 81

Golubaev, A. V. 2015, SoSyR, 49, 147

Jopek, T. J. \& Jenniskens, P. M. 2011, in Meteoroids: The Smallest Solar System Bodies, ed. W. J. Cooke, D. E. Moser, B. F. Hardin, \& D. Janches, 7

Jopek, T. J. \& Kaňuchová, Z. 2014, The Meteoroids 2013, eds. J. J. Jopek, F. J. M. Rietmeijer, J. Watanabe, \& I. P. Williams (A. M. Univ. Press), 353

Karpov, S., Beskin, G., Biryukov, A., et al. 2005, NCmiC, 28, 747

Karpov, S., Beskin, G., Bondar, S., et al. 2010, AdAst, 2010

Karpov, S., Katkova, E., Beskin, G., et al. 2016a, RMxAC, 48, 112

Karpov, S., Orekhova, N., Beskin, G., et al. 2016b, RMxAC, 48, 97

Karpov, S., Beskin, G., Biryukov, A., et al. 2017, ASPC, 510, 309

Koukal, J., Srba, J., \& Gorková, S. 2015, in International Meteor Conference Mistelbach, Austria, ed. J.L. Rault \& P. Roggemans (Austria), 91

SonotaCo. 2009, WGN, JIMO, 37, 55

Vallado, D. 2007, Fundamentals of Astrodynamics and Applications (Berlin: Springer), 150

Zolotukhin, I., Beskin, G., Biryukov, A., et al. AN, 325, 675

\footnotetext{
${ }^{7}$ http://mmt.favor2.info/static/meteors_annual_ activity.gif
} 